\section{SAT0638 CLINICAL ACTIVITY, ULTRASOUND ASSESSMENT AND DRUG MONITORING IN RHEUMATOID ARTHRITIS PATIENTS RECEIVING ANTI-TNF- $\alpha$ THERAPY WITH EXTENDED INTERVAL OF ADMINISTRATION}

J.M. Senabre Gallego ${ }^{1}$, J. Rosas Gómez de Salazar ${ }^{1}$, M. Marco Mingot ${ }^{2}$, A. Naranjo ${ }^{3}$, F. Llinares-Tello ${ }^{2}$, A. Pons ${ }^{1}$, X. Barber-Vallés ${ }^{4}$, G. Santos-Soler ${ }^{1}$, E. Salas-Heredia ${ }^{1}$, C. Cano ${ }^{1}$, M. Lorente ${ }^{1}$, J.A. García Gómez ${ }^{4}$, J. Molina ${ }^{2}$ on behalf of Asociación para la Investigación en Reumatología de la Marina Baixa (AIRE-MB). ${ }^{1}$ Rheumatology; ${ }^{2}$ Laboratory, Hospital Marina Baixa, Villajoyosa (Alicante); ${ }^{3}$ Rheumatology, Hospital Dr. Negrín, Las Palmas de Gran Canaria; ${ }^{4}$ Centro de Investigación Operativa, Universidad Miguel Hernández, Elche, Spain

Objectives: To assess clinical activity, ultrasound synovitis and drug levels in rheumatoid arthritis (RA) patients receiving anti-TNF $\alpha$ therapy with extended interval of administration (EIA).

Methods: Prospective observational study. Population: RA patients, in clinical remission, receiving adalimumab (ADL) or etanercept (ETN) with EIA. Clinical activity was assessed by DAS28-ESR, DAS28-CRP, CDAl and SDAl scores at each visit. Twelve-joint ultrasound assessment (elbows, wrists, 2nd and 3rd metacarpophalangeal joints, knees and ankles) was performed evaluating synovitis through B-mode (BM) and Color Doppler signal (CD). A BM and CD score was calculated summing the highest score from each joint to a maximum of 36 points. We consider positive score $>1$ point. Serum drug levels were measured using Promonitor ${ }^{\circledR}$ ELISA kits (Progenika Biopharma-Grifols, Spain).

Results: A total of 39 patients were included since February 2011 to December 2016. One patient was excluded due to blindness violation and 2 patients never reduced anti-TNF $\alpha$ due to low drug levels. 31 patients were women (82\%) and the mean age was 61 (39-81) years. Most patients were RF positive (87\%) and ACPA positive (74\%). 22 patients were with ADL treatment and 16 with ETN. 32 patients (82\%) were with DMARD concomitant treatment (18 MTX (46\%), 11 LEF (18\%), 2 HCQ (5\%), 1 SSZ (2\%)) and 7 patients were with low-dose CS (18\%). Mean time from diagnosis was 14,95 years (range 2,15-52,31) and Mean time with current biologic drug was 4,21 years (range $1,39-11,07$ ). Nine patients $(24 \%)$ returned to standard interval due to worsening of clinical activity and one discontinued treatment due to septic arthritis. All of them returned to clinical remission and no anti-drug antibodies were detected. Clinical activity scores, ultrasound scores and drug levels are summarized in table 1.

Table 1. Clinical activity scores, ultrasound scores and drug levels

\begin{tabular}{lccc}
\hline & Basal visit & 6 months & 12 months \\
\hline $\mathrm{N}$ & 32 & 31 & 20 \\
DAS28-ESR & $2,0(0,91)$ & $1,83(0,83)$ & $1,61(0,70)$ \\
DAS28-CRP & $1,71(0,51)$ & $1,78(0,52)$ & $1,62(0,48)$ \\
SDAl & $4,25(2,55)$ & $4,27(3,04)$ & $3,64(3,13)$ \\
CDAl & $3,82(2,48)$ & $3,61(2,33)$ & $2,76(1,3)$ \\
BM score & $3,57(4,57)$ & $3,29(3,81)$ & $4,7(4,32)$ \\
CD score & $0,78(0,80)$ & $1,19(1,7)$ & $1,15(1,42)$ \\
BM score (\%) & 73,91 & 77,42 & 90,00 \\
CD score (\%) & 56,52 & 45,16 & 50,00 \\
ETN & $4,61(2,74$ & $3,59(2,9)$ & $3,03(0,84)$ \\
ADL & $12,05(6,88)$ & $8,49(5,01)$ & $6,49(2,78)^{*}$ \\
\hline${ }^{*} \mathrm{P}<0,05$. All results mean $(S D)$ otherwise specified. & &
\end{tabular}

Conclusions: 1. Clinical remission was sustained in most patients receiving ADL or ETN in extended interval of administration (EIA). 2. Drug levels decrease over time. 3 . Some patients $(24 \%)$ returned to standard interval of administration due to clinical worsening. 4. Some patients show subclinical ultrasound synovitis in B-mode ( $90 \%$ ) or Color Doppler (50\%) 5 . It would be advisable to perform periodic ultrasound and monitoring of anti-TNF $\alpha$ levels to maintain clinical remission in patients with EIA.

Acknowledgements: This study was supported by a research grant from Fundación Española de Reumatología and AIRE-MB.

Disclosure of Interest: J. Senabre Gallego Speakers bureau: MSD, Novartis, Abbvie, UCB, BMS, J. Rosas Gómez de Salazar: None declared, M. Marco Mingot: None declared, A. Naranjo: None declared, F. Llinares-Tello: None declared, A. Pons: None declared, X. Barber-Vallés: None declared, G. Santos-Soler: None declared, E. Salas-Heredia: None declared, C. Cano: None declared, M. Lorente: None declared, J. A. García Gómez: None declared, J. Molina: None declared DOI: 10.1136/annrheumdis-2017-eular.3066

\section{SAT0639 BIOLOGIC-ELIGIBLE RHEUMATOID ARTHRITIS PATIENTS DEMONSTRATE ABSENCE OF SYNOVITIS - UTILITY OF ULTRASOUND IN THE MANAGEMENT OF RA-BIOLOGIC POPULATION}

K. Naraghi $^{1}$, T. Vojinovic ${ }^{2}$, J. Nam ${ }^{1}$, J. Freeston ${ }^{1}$, P. Emery ${ }^{1}$, R.J. Wakefield ${ }^{1}$, M.H. Buch ${ }^{1}$. ${ }^{1}$ Leeds Institute of Rheumatic \& Musculoskeletal Medicine, University of Leeds, Leeds, United Kingdom; ${ }^{2}$ Faculty of Medicine and Surgery, University of Pavia, Pavia, Italy

Background: Biologic drugs act by suppressing synovitis in patients with rheumatoid arthritis (RA). Clinical disease activity scores (DAS) are used to determine eligibility and assess biologic drug response. However, several drivers aside from synovitis may drive the DAS. Joint ultrasound (US) scan is more accurate than clinical examination in detecting inflammatory synovitis [1, 2], and is frequently used in clinical practice to assess need for biologic drug change [3]. Objectives: To phenotype DAS using US in patients eligible for a first/switch in biologic therapy and to evaluate whether and how this changed decision making. Methods: A retrospective evaluation of RA patients who attended our tertiary centre biologic clinic between 2014 and 2016 and had an US to clarify basis for raised disease activity. Three-variable DAS28-CRP 28 joints (DAS28-CRP) was recorded and US reports were evaluated to determine which of the following characteristics were observed: presence of synovitis, tenosynovitis, osteoarthritis, tendinopathy and no abnormality. Clinic notes were reviewed to record change in the management of patients as a result of combined clinical and US scan findings. Results: We identified 70 patients who had not received steroids within 4 weeks of the US, $84.3 \%$ female, median (range) age 58 (20-88) years. 66 $(94.3 \%)$ patients were biologic experienced with 59 on ongoing treatment [3 (5.1\%) abatacept, $22(37.3 \%)$ anti-TNF $\alpha, 22(37.3 \%)$ rituximab and $12(20.3 \%)$ tocilizumab] of whom $47(79.7 \%)$ were on combination with csDMARD therapy. 4 (5.7\%) patients were biologic naive. $42(63.6 \%)$ of bio-experienced patients, had a DAS28 $>3.2$ and were eligible for a change in their biologic however, only 17 of these patients had US-confirmed synovitis, 21 had evidence of osteoarthritis $(\mathrm{OA}), 1$ tenosynovitis and 3 had no abnormalities. All biologic naïve patients had DAS28-CRP $\leq 5.1$ before US however, 3 had US-confirmed synovitis and 1 had OA. Of bio-experienced, 13/66 (19.4\%) patients, including 4 with DAS-28 $<3.2$ but US-confirmed synovitis, had a new biologic started. 3 rituximab patients (including one with subclinical synovitis) had re-treatment. Concomitant treatment was escalated in $13(24.2 \%)$ patients. $35(53.0 \%)$ patients, including 4 with US synovitis/tenosynovitis, had no change in their treatment. 2 (3.0\%) patients self-discontinued treatment.

Conclusions: By identifying lack of inflammatory synovitis in biologic eligible patients US reduced the need for unnecessary and costly change of biologics. US also detected subclinical synovitis warranting modification of their treatment.

References:

[1] Colebatch AN, Edwards CJ, Ostergaard M, et al. EULAR recommendations for the use of imaging of the joints in the clinical management of rheumatoid arthritis. Annals of the rheumatic diseases. 2013; 72:804-814.

[2] Saleem B, Brown AK, Keen $\mathrm{H}$, et al. Should imaging be a component of rheumatoid arthritis remission criteria? A comparison between traditional and modified composite remission scores and imaging assessments. Annals of the rheumatic diseases. 2011; 70:792-798.

[3] lagnocco A, Finucci A, Ceccarelli F, et al. Power Doppler ultrasound monitoring of response to anti-tumour necrosis factor alpha treatment in patients with rheumatoid arthritis. Rheumatology (Oxford). 2015; 54:1890-1896.

Disclosure of Interest: None declared

DOI: 10.1136/annrheumdis-2017-eular.6633

\section{SAT0640 DIAGNOSTIC UTILITY OF DETECTING ENTHESITIS BY ULTRASOUND IN PERIPHERAL SPONDYLOARTHRITIS}

K. Fujikawa ${ }^{1}$, Y. Endo ${ }^{1}$, A. Mizokami ${ }^{1}$, M. Mine ${ }^{2}$, A. Kawakami ${ }^{3}$ ${ }^{1}$ Rheumatology, Japan Community Healthcare Organization, Isahaya General Hospital; ${ }^{2}$ Rheumatology, Suga Orthopedic Hospital, Isahaya; ${ }^{3}$ Department of Immunology and Rheumatology, Graduate School of Biomedical Sciences, Nagasaki University, Nagasaki, Japan

Objectives: To evaluate the diagnostic utility of detecting enthesitis by ultrasound in patients with peripheral spondyloarthritis $(\mathrm{SpA})$.

Methods: A single-center cohort study was performed in patients with symptoms suggestive of peripheral SpA (inflammatory back pain, arthritis of the lower limbs, tenderness of the entheses and dactylitis). Patients with only axial symptoms in the absence of peripheral symptoms were excluded. Fourteen sites of enthesis (both sides of lateral epicondyle, quadriceps tendon insertion into the patella, patellar ligament insertion into the patella and tibial tuberosity, medial and lateral femoral condyles, and Achilles tendon) were assessed at baseline by ultrasound. Ultrasound assessment was made by Japan College of Rheumatology (JCR)registered sonographers. Furthermore, articular synovitis and tenosynovitis of both wrist and finger joints as well as symptomatic sites were examined. Clinical, laboratory (CRP, HLA typing), radiological (X-ray and MRI of sacroiliac joint) findings and SpA classification criteria (Amor's, ESSG and ASAS) were also evaluated. The gold standard was the diagnosis made by the JCR-certified rheumatologists during a six-month follow-up period.

Results: Between April 2014 and November 2016, one hundred-thirty six patients were consecutively enrolled. A definite diagnosis was obtained in 112 patients (72 SpA and 40 non-SpA). Diagnosis was not made in the remaining 24 patients. Seventy-two SpA patients ( 62 with undifferentiated SpA, 6 with psoriatic arthritis, 2 with AS, 1 with inflammatory bowel disease-associated arthritis and 1 with reactive arthritis) and 40 non-SpA patients were investigated in this study. In ultrasound findings, SpA patients showed power Doppler (PD) signals of the articular synovium $(57 \%)$, tendon sheath synovium $(71 \%)$ and enthesis $(94 \%)$. A PD signal for at least one enthesis sites was the most useful finding for differentiation of SpA from non-SpA (sensitivity $94 \%$; specificity $85 \%$; accuracy $91 \%$; positive likelihood ratio 6.3) regarding ultrasound findings. In logistic regression analysis, fulfillment of peripheral ASAS criteria, that of Amor's criteria, and presence of PD signals at least one enthesis sites were independent variables to the contribution of diagnosis of SpA (Figure 1). 